\title{
An enhanced immunocytochemical method for staining bone marrow trephine sections
}

\author{
W N Erber, J I Willis, G J Hoffman
}

\begin{abstract}
Aims-The detection of cellular antigens in fixed decalcified bone marrow trephine (BMT) sections depends on the method of processing, the nature of the antigen and antibody, antigen retrieval techniques, and the sensitivity of the immunocytochemical method. This study evaluated a tyramide enhanced avidin-biotin immunostaining method on formalin fixed decalcified BMT sections to determine whether the method could detect previously undetectable antigens.

Methods-Nineteen BMT biopsies from a range of haematological disorders were evaluated with 43 antibodies to haemopoietic antigens using horseradish peroxidase and alkaline phosphatase detection methods, using the tyramide enhanced avidin-biotin immunostaining method.

Results-Compared with standard avidin-biotin immunostaining methods the tyramide enhanced immunostaining method showed enhanced signal intensity, gave positive labelling for antigens that require pretreatment by other methods, and previously unreactive antigens were detected. Primary antibodies could be used at up to 200 times higher dilutions.

Conclusion-The tyramide enhanced immunostaining method, while retaining specificity, is highly sensitive and enables an increased number and range of antigens to be detected than previously possible. The method could be applied to BMT sections for the routine diagnosis and classification of haematological disorders. (f Clin Pathol 1997;50:389-393)
\end{abstract}

Haematology

Department, Royal

Perth Hospital, Box

X2213 GPO Perth,

Western Australia

6001, Australia

W N Erber

J I Willis

Western Diagnostic Pathology, 74 McCoy

Street, Myaree,

Western Australia

6154, Australia

G J Hoffman

Correspondence to:

Dr Wendy N Erber,

e-mail: wenderbe@

dunamis.rph.uwa.edu.au

Accepted for publication 25 February 1997
Keywords: bone marrow trephines; immunocytochemistry; tyramide

Bone marrow trephine (BMT) biopsies are particularly valuable for the assessment of haematological diseases where there has been a failure to aspirate bone marrow, the disease is not readily amenable to examination in the bone marrow aspirate, or to assess focal disease. $^{1-2}$ The immunocytochemical evaluation of BMT sections is used for the classification of lymphomas and leukaemias, and for monitoring residual disease. ${ }^{3-8}$ However, immunocytochemical techniques have been limited by the denaturing or masking of some tissue antigens as a result of the formalin fixation, decalcification, and tissue processing. Antigen retrieval methods using enzymes (such as pronase or trypsin), microwave oven heating or pressure cooking ${ }^{9}{ }^{10}$ have increased the range of antigens detected in BMT sections. ${ }^{11}$ However, the method is still suboptimal detecting fewer antigens than in fresh cell smears or tissue sections. ${ }^{11-15}$

Antigenicity is dependent not only on tissue processing and antigen retrieval but also on the sensitivity of the immunocytochemical method applied. ${ }^{16}$ The majority of studies have used indirect antibody staining methods, unlabelled antibody bridge methods (for example, alkaline phosphatase anti-alkaline phosphatase) and avidin-biotin enzyme labelled methods; the last having the greatest sensitivity. Recently the avidin-biotin system has been further sensitised by the use of biotinyl tyramide, a phenolic compound. ${ }^{16-19}$ This detection method is based on standard avidin-biotin technology: primary antibody incubation, followed by biotinylated antimouse immunoglobulin and streptavidinbiotin-horseradish peroxidase complexes. Staining enhancement is achieved by incubation with biotinylated tyramide which binds covalently to electron rich moieties at the active sites of horseradish peroxidase. ${ }^{16}$ The biotin molecules coupled to the tyramide bind further horseradish peroxidase labelled streptavidin, increasing the intensity of the reaction product over standard methods. It has been claimed that the biotinylated tyramide results in a 100 to 10000 -fold increase in sensitivity without loss of specificity, and that primary antibodies can be used at dilutions 500 times higher than with standard avidin-biotin techniques. ${ }^{18}$ In addition it has been reported that this method enables antibodies that were previously unreactive in formalin fixed paraffin wax embedded tissue to give positive immunostaining. ${ }^{19}$

The aim of the present study was to establish whether the biotinylated tyramide enhancement immunostaining (TEI) method gave significant enhancement in BMT sections and enabled additional antigens to be detected. We have explored the utility of this biotinylated tyramide enhancement in horseradish peroxidase and alkaline phosphatase detection systems on BMT biopsy sections from a range of haematological disorders. Forty three antibodies were assessed, and the reactivity using the TEI method compared with the intensity of staining achieved with a standard avidin-biotin immunoalkaline phosphatase technique and two antigen retrieval methods.

\section{Materials and methods}

SPECIMENS

BMT biopsies for routine diagnosis obtained from patients with a range of haematological 
Table 1 Details of the bone marrow trephine biopsies assessed by the biotinylated tyramide enhanced immunostaining method

\begin{tabular}{lc}
\hline Disorder & Number of cases \\
\hline Normal bone marrow & 2 \\
Acute lymphoblastic leukaemia & 2 \\
Acute myeloid leukaemia & 3 \\
$\quad$ Acute myeloblastic leukaemia (M2) & 1 \\
Acute myelomonocytic leukaemia (M4) & 1 \\
Acute megakaryoblastic leukaemia (M7) & 1 \\
Chronic lymphocytic leukaemia & 2 \\
Hairy cell leukaemia & 1 \\
Non-Hodgkin's lymphoma & 6 \\
Follicular small cleaved cell & 2 \\
Diffuse mixed small and large cell & 1 \\
Diffuse large cell & 2 \\
Mantle cell & 1 \\
Hodgkin's disease & 1 \\
Multiple myeloma & 2 \\
Total & 19
\end{tabular}

disorders were studied (table 1). The biopsies were fixed in formol acetic acid for four to six hours, decalcified in formic acid for three to six hours and embedded in paraffin wax-the standard BMT processing protocol for our institution. ${ }^{11}$ Sections were cut at 3-5 $\mu \mathrm{m}$ and mounted on aminosilane coated slides.

MONOCLONAL ANTIBODIES

A total of 43 monoclonal and polyclonal antibodies to antigens expressed by haemopoietic cells were assessed (table 2). Many of the antibodies assessed were known not to detect antigens in BMT sections by standard immunocytochemical techniques. The antibodies were directed against lymphoid, myeloid, erythroid, megakaryocytic, and non-lineage associated antigens. All the primary antibodies used in the study were from Dako A/S (Glostrup, Denmark) except: CD5/54 (CD5), Y-2/178 (CD5), X1-7 (CD8) and VS5e (CD13) which were generously provided by $\mathrm{D}$ Mason (Oxford, UK); BerAct8 (CD103) from H Stein (Berlin, Germany); HuLym2 (CD7) from I McKenzie (Melbourne, Australia); and My4 (CD14) from Coulter Electronics (Australia).

\section{BIOTINYLATED TYRAMIDE ENHANCED}

IMMUNOSTAINING

BMT sections were de-waxed twice in xylene and acetone and rehydrated in Tris buffered saline (TBS; $0.05 \mathrm{M}, \mathrm{pH} 7.6$ ). The dewaxed sections were incubated with $3 \%$ hydrogen peroxide for five minutes to block endogenous peroxidase followed by a five minute protein blocking step. No antigen retrieval was performed for sections stained by the TEI method. Primary antibody was applied to the BMT sections for 30 minutes at room temperature and then washed in TBS. Some primary antibodies (monoclonal antibodies $\mathrm{P}-1 / 33$ (CD9) and $\mathrm{Y}-2 / 51$ (CD61), and polyclonal anti-kappa and lambda) were tested at a range of dilutions. Slides stained with polyclonal antibodies were then incubated with mouse antirabbit immunoglobulin (Dako M737). Immunostaining was performed using the Dako Catalysed Signal Amplification system (Dako K1500). In brief, biotinylated antimouse immunoglobulin was applied for 15 minutes followed by streptavidin-biotin- horseradish peroxidase complex. The sections were washed, incubated with biotinylated tyramide for 15 minutes, washed, and streptavidinhorseradish peroxidase added. After washing, diaminobenzidine substrate and hydrogen peroxide were added and incubated for five minutes. In some experiments the final streptavidin-horseradish peroxidase step was replaced by streptavidin labelled alkaline phosphatase (Dako K687), and fast red naphtholAS-MX phosphate used as the substrate. Slides were counterstained with haematoxylin and coverslips mounted prior to light microscopic examination.

AVIDIN-BIOTIN ALKALINE PHOSPHATASE STAINING BMT sections were dewaxed and antigen retrieval (microwave heating or enzyme digestion) performed for some antibodies prior to application of the primary antibody. Microwave heating was performed by placing the de-waxed slides in a plastic Coplin jar containing a boiling solution of $0.01 \mathrm{M}$ tri-sodium citrate and covered. The slides were irradiated in a standard domestic microwave $(650 \mathrm{~W})$ on high for two minutes and medium for eight minutes. The buffer solution was maintained above the level of the BMT section throughout the microwave heating. The slides were left to cool in the citrate buffer for 10 minutes before rinsing in TBS. Proteolytic enzyme digestion was performed in a Coplin jar containing trypsin solution $(0.1 \%$ in TBS containing calcium chloride) at $37^{\circ} \mathrm{C}$ for 15 minutes. Sections were washed in TBS prior to application of the primary antibody.

The primary antibody was incubated, followed by mouse antirabbit immunoglobulin for polyclonal antibodies, and the sections stained using the labelled avidin-biotin alkaline phosphatase immunocytochemical method (Dako K687). In brief this method involves addition of biotinylated antimouse immunoglobulin and subsequent incubation with alkaline phosphatase labelled avidin and fast red naphtholAS-MX phosphate substrate. The sections were counterstained with haematoxylin, a cover slip mounted, and examined by light microscopy.

A qualitative comparison was made of the intensity of positive labelling by the avidinbiotin alkaline phosphatase and the tyramide enhanced immunostaining (TEI) system.

\section{Results}

The results of the immunocytochemical analysis are summarised in tables 2 and 3. Strong staining specific for antigen positive cells was achieved. The appropriate localisation of staining was seen-for example, cell membrane staining (such as CD20), cytoplasmic (such as bcl-2) or nuclear (such as TdT) depending on the antigen specificity. Marrow architecture and cytological detail were well preserved and there was no non-specific background cellular or intercellular staining. The staining was reproducible and identical patterns of staining and intensity were obtained with horseradish peroxidase and alkaline phosphatase detection methods. 
Table 2 Antibody reactivity with the tyramide enhanced immunostaining method

\begin{tabular}{llll}
\hline Group 1 & Group 2 & Group 3 & Group 4 \\
\hline CD6 (ST 23) & CD5 (CD5/54) & Bcl-2 (Bcl-2/100) & CD2 (T9-10) \\
CD9 (P-1/33) & CD7 (HuLym 2) & CD20 (L26) & CD3 (UCHT1) \\
CD11a (MHM24) & CD8 (C8/144) & CD31 (JC70a) & CD4 (T3/10) \\
CD16 (DJ130) & CD30 (Ber H2) & CD43 (DFT1) & CD5 (Y-2/178) \\
CD22 (4KB128) & CD61 (Y-2/51) & HLA-DR (CR3/43) & CD8 (X1-7) \\
CD23 (MHM6) & CD79a (JCB117) & HLA-DR (TAL1B5) & CD10 (SS-2/36) \\
CD33 (WM54) & R $\alpha$ kappa & R $\alpha$ CD3 & CD11b (2LPM19c) \\
CD68 (Y-1/82a) & R $\alpha$ lambda & & CD11c (KB90) \\
& R $\alpha$ TdT & & CD13 (VS5e) \\
& & & CD14 (MY-4) \\
& & & CD19 (HD37) \\
& & & CD25 (ACT-1) \\
& & & CD103 (BerAct 8) \\
& & & Ki67 \\
& & & MPO (MPO-7) \\
& & & Kappa (A8B5) \\
& & & Lambda (N10/2) \\
& & & RER (RFD6) \\
& & & Vimentin (V9) \\
\end{tabular}

Group 1, TEI positive/AB-AP negative (with and without pretreatment); Group 2, TEI positive AB-AP with pretreatment positive; Group 3, TEI and AB-AP positive/TEI enhanced; Group 4, TEI and AB-AP negative.

TEI, biotinylated tyramide enhancement immunostaining; AB-AP, avidin-biotin alkaline phosphatase; $R \alpha$, rabbit polyclonal antibody.

Table 3 Comparison of antibody reactivity of the biotinylated tyramide enhanced immunostaining method with the avidin-biotin alkaline phosphatase method

\begin{tabular}{llll}
\hline $\begin{array}{l}\text { Antibody } \\
\text { groups }\end{array}$ & $\begin{array}{l}\text { Tyramide enhanced } \\
\text { immunostaining (TEI) }\end{array}$ & $\begin{array}{l}\text { Avidin-biotin (AB) AP } \\
\text { with antigen retrieval }\end{array}$ & $\begin{array}{l}\text { Avidin-biotin AP without } \\
\text { antigen retrieval }\end{array}$ \\
\hline Group 1 & +++++ & - & - \\
Group 2 & +++ & ++ & - \\
Group 3 & +++ & ++ & + \\
Group 4 & - & - & - \\
\hline
\end{tabular}

Group 1, TEI positive/AB-AP negative (with and without pretreatment); Group 2, TEI positive/ AB-AP with pretreatment positive; Group 3, TEI and AB-AP positive/TEI enhanced; Group 4, $\mathrm{TEI}$ and $\mathrm{AB}-\mathrm{AP}$ negative.

++++ , intensity of positive staining; + , weak staining; ++ , moderate staining; +++ , strong staining; -negative.

AP, alkaline phosphatase.

When results of staining with the tyramide enhancement system were compared with the standard avidin-biotin immunoalkaline phosphatase technique used routinely in the authors' laboratory, significant differences between the two methods were seen. The antibody reactivity patterns could be classified into four groups based on these differences (tables 2 and 3).

TEI POSITIVE, AVIDIN-BIOTIN ALKALINE PHOSPHATASE NEGATIVE

Eight antibodies (for example, CD9, CD16, CD23) giving no staining by the conventional avidin-biotin alkaline phosphatase method (either with or without antigen retrieval methods) on fixed decalcified BMT showed positive staining with the TEI method. The antibodies stained the appropriate cells in normal and leukaemic BMTs. For example, CD9 (figs 1 and 2) stained megakaryocytes, plasma cells, eosinophils, and the blast cells in common acute lymphoblastic leukaemia; CD16 stained granulocytic cells; and CD23 the lymphocytes in B cell chronic lymphocytic leukaemia.

\section{TEI POSITIVE, AVIDIN-BIOTIN POSITIVE WITH} MICROWAVE OR ENZYME PRETREATMENT Nine antibodies (for example, CD30, CD61, CD79a) that require enzyme or microwave antigen retrieval in the standard avidin-biotin alkaline phosphatase method gave positive staining by the TEI system without pretreatment (fig 3). The cytological preservation was superior in the TEI method as a consequence, and the intensity of the signal was significantly enhanced.

TEI ENHANCED INTENSITY COMPARED WITH AVIDIN-BIOTIN ALKALINE PHOSPHATASE This group of antibodies do not require pretreatment for conventional avidin-biotin staining but gave significantly enhanced signal intensity with TEI compared with the conventional avidin-biotin alkaline phosphatase method (for example, bcl-2, polyclonal CD3; fig 4).

NO REACTIVITY BY TEI OR AVIDIN-BIOTIN

ALKALINE PHOSPHATASE

Nineteen of the forty three antibodies remained unreactive by both the amplified TEI method and the standard avidin-biotin alkaline phosphatase method.

There were no antibodies that were positive with the conventional avidin-biotin method (with or without pretreatment) and negative with the TEI system. Titrations of primary antibodies showed that dilutions greater than those used for the standard alkaline phosphatase method still yielded maximal staining intensity. For example, polyclonal kappa and lambda antibodies could be diluted an additional 1:100, while P-1/33 (CD9) and Y-2/51 (CD61) an additional 1:200.

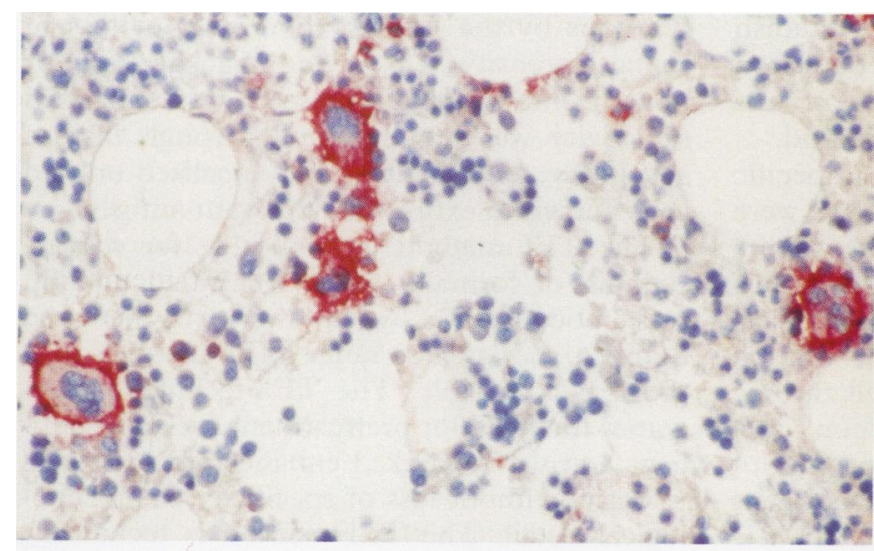

Figure 1 Antibody P-1/33 (CD9) staining of a normal bone marrow trephine by the TEI method and alkaline phosphatase detection (fast red substrate). Note the strong positive staining of the megakaryocytes (original magnification $\times 160$ ).

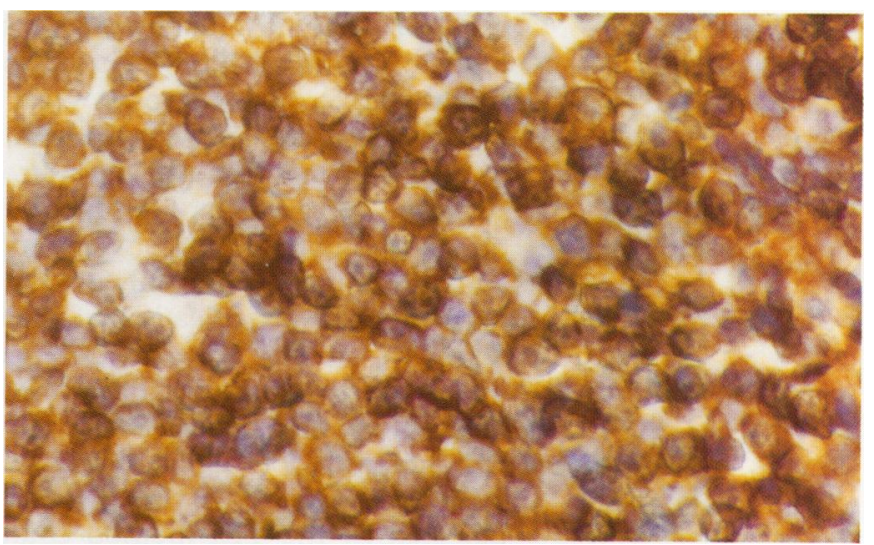

Figure 2 Bone marrow trephine section from a case of common acute lymphoblastic leukaemia stained with P-1/33 (CD9) by the TEI method. The majority of blast cells are positive. Horseradish peroxidase and diaminobenzidine detection (original magnification $\times 400$ ). 


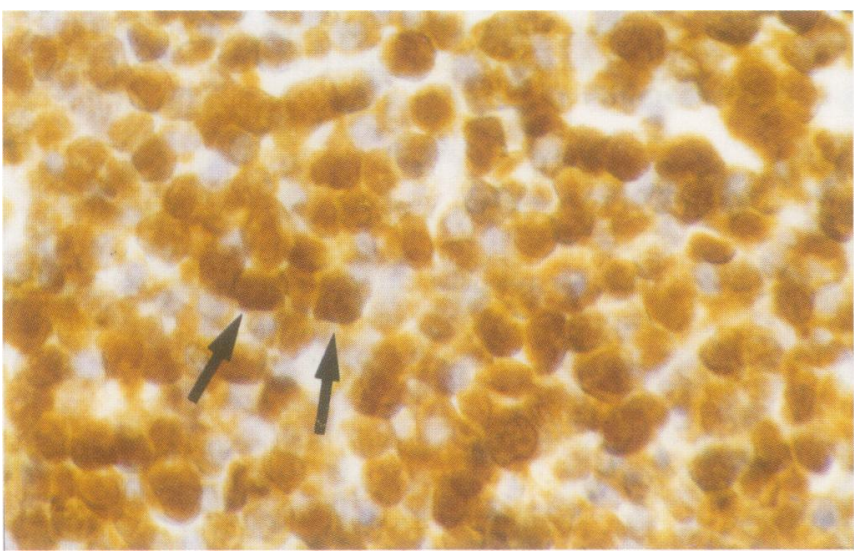

Figure 3 Bone marrow trephine section from a case of common acute lymphoblastic leukaemia stained for terminal deoxynucleotidyl transferase (TdT) by the TEI method. Note the strong nuclear staining of the positive blast cells (representative positive cells arrowed). (Horseradish peroxidase and diaminobenzidine detection, original magnification $\times 400$ ).

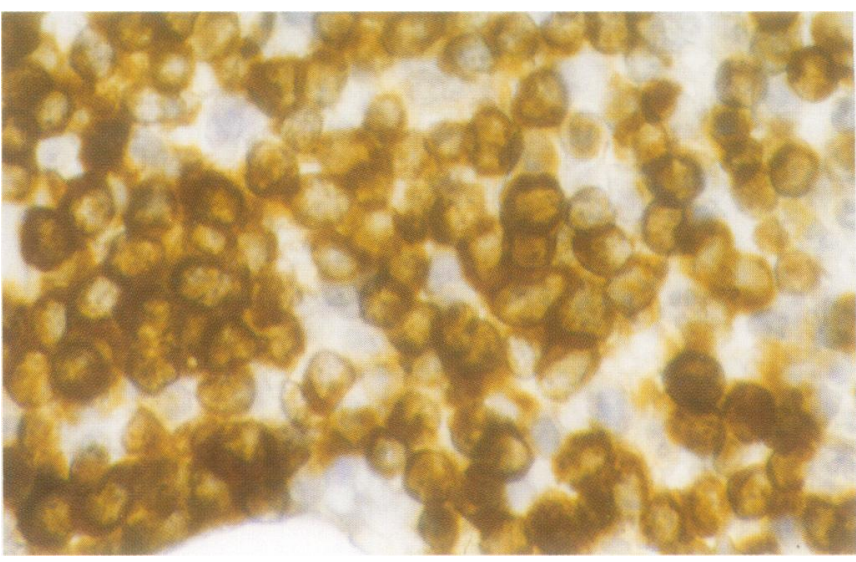

Figure $4 T$ cell acute lymphoblastic leukaemia stained for $\mathrm{Ra} C D 3$ (polyclonal rabbit anti-CD3) by the TEI method. The majority of lymphoblasts are positive. (Horseradish peroxidase and diaminobenzidine detection, original magnification $\times 400$ ).

\section{Discussion}

Immunocytochemical evaluation of BMT sections has significantly aided the conventional histological examination of BMT biopsies. However, limitations have been experienced due to fixation, decalcification, and tissue processing reducing antigenicity. Antigen retrieval methods (for example, enzyme pretreatment, microwave heating) have broadened the number and range of antigens detectable on BMT sections for the study of haematological disorders. ${ }^{811}$ However, several antigens of relevance to diagnostic haematopathology remain undetectable. In addition, antigen retrieval methods must be individualised for each antibody, making this method of staining cumbersome. Also, cytological distortion may occur as a result of pretreatment methods. ${ }^{11}$ Hence an enhanced immunocytochemical detection system that does not require antigen retrieval would be a major benefit.

The results of the present study indicate that the obstacles resulting from BMT processing can be overcome for many antigens by using a TEI system. This method gave significantly enhanced intensity of the staining reaction over the standard immunoalkaline phosphatase method used in the authors' laboratory and enabled a much broader range of antigens to be detected. The results obtained were reproducible and removed the need for antigen retrieval pretreatment. In addition the method can be used for immunostaining by both horseradish peroxidase and alkaline phosphatase detection systems. Excellent morphological detail was consistently obtained with the TEI method.

The TEI method also gave strong specific staining of antigens in BMT that were previously undetectable. Many of these are antigens that are commonly used in the immunophenotypic classification of haematological disorders in cell smears or frozen section material-for example, CD23 in B cell chronic lymphocytic leukaemia; CD9 in common acute lymphoblastic leukaemia and acute promyelocytic leukaemia. ${ }^{20-22}$ The range of antigens detected by the TEI method could make it a useful technique in classifying haematological malignancies in BMT sections. For example, acute leukaemias could be divided into com- mon acute lymphoblastic leukaemia (CD9, CD79a, TdT positive), $T$ cell acute lymphoblastic leukaemia (CD3, CD7, TdT positive), and acute myeloid leukaemia (CD33, CD68 positive) in BMT sections, and many of the chronic lymphoproliferative disorders classified with CD5, CD6, and CD23 antibodies. ${ }^{20}$

The biotinylated tyramide enhancement system therefore enables a number of antibodies that were previously ineffective on decalcified BMT sections to give positive staining. The ability to detect previously undetectable antigens, such as CD9, CD16, and CD23, by the TEI method indicates that these antigens have not been destroyed by the BMT processing, but "masked" and require a highly sensitive detection method. Some antigens still remain undetectable, such as CD11c, and CD25, despite reports of positive staining for these antigens by the. TEI method in other fixed tissues. ${ }^{19}$ This suggests that the decalcification process has an additional effect over that of formalin fixation on some antigens in BMT sections, resulting in denaturation or "masking".

A number of antibodies that previously only gave positive signals with antigen retrieval methods, such as CD30, CD61, and CD79a, gave positive signals using the TEI method without the need for pretreatment. Further, the intensity of the labelling was greater and primary antibodies could be used at higher dilutions by the TEI method without loss of signal intensity. ${ }^{18} 19$ As no background or non-specific staining was seen, this enhanced sensitivity was not achieved through loss of specificity; the positive signal localised only to cells known to express the relevant antigen.

The TEI method is applicable for routine staining of formalin fixed decalcified paraffin wax embedded BMT sections, with an increased number and greater range of antibodies than previously possible. The TEI technique overcomes the need for pretreatment for many antigens and yields marked enhancement of signal intensity without loss of specificity or morphological detail. The TEI method is technically simple but is more time consuming than other avidin-biotin methods. The range of antigens that can be detected with the TEI system would 
enable it to be applied to BMT sections for the diagnosis and classification of the majority of leukaemias and lymphomas, monitoring residual malignant disease, and detection of early relapse of disease involving the marrow. Some diagnostically useful antigens, such as CD11c and CD25 in hairy cell leukaemia, and CD10 in common acute lymphoblastic leukaemia, remain undetectable.

The authors thank Sharon Holt and Karen Wright for their secretarial assistance in the preparation of the manuscript.

1 Brown DC and Gatter KC. The bone marrow trephine biopsy: A review of normal histology. Histopathology biopsy: A revie

2 Frisch B, Lewis SM, Burkhardt R, Bartl R. Biopsy pathology of bone and bone marrow. New York: Raven Press, 1985.

3 Gatter KC, Heryet A, Brown DC, Mason DY. Is it necessary to embed bone marrow biopsy in plastic for haematological diagnosis? Histopathology 1987;11:1-7.

4 Erber WN, McLachlan JM. Use of the APAAP technique on paraffin wax-embedded bone marrow trephines. $\mathcal{f}$ Clin Pathol 1989;42:1201-5.

5 Van der Valk P, Mullinck H, Huijgens PC, Tadema TM, Vos W, Meijer CILM. Immunohistochemistry in bone marrow diagnosis. Value of a panel of monoclonal antibodies on diagnosis. Value of a panel of monoclonal antibodies on routinely processed bo

6 Thaler J, Dietze O, Denz H, Demuth R, Nachbaur D, Stauder R, et al. Bone marrow diagnosis in lymphoproliferative disorders: Comparison of results obtained from conventional histomorphology and immunohistology. Histopathology 1991;18:495-504.

7 Horny H-P, Wehrmann M, Schlicker HUH, Eichstaedt A, Clemens MR, Kaiserling E. QBEND-10 For the diagnosis of myelodysplastic syndromes in routinely processed bone marrow biopsy specimens. $\mathcal{f}$ Clin Pathol $1995 ; 48: 291-4$.

8 Orazi A, Cotton J, Cattoretti J, Kotylo PK, John K, Manning JT, et al. Terminal deoxynucleotidal transferase staining in acute leukaemia and normal bone marrow in routinely processed paraffin sections. Am $\mathcal{f}$ Clin Pathol 1994;102: $640-5$

9 Bankfalvi A, Navabi H, Bier B, Börcker W, Jasani B, Schmid $\mathrm{KW}$. Wet autoclave pre-treatment for antigen retrieval in diagnostic immunohistochemistry. F Pathol 1995;174:223-8.
10 Miller K, Auld J, Jesseb E, Rhodes A, Ashton-Key M. Antigen unmasking in formalin-fixed routinely processed paraffin wax-embedded sections by pressure cooking: a comparison with microwave oven heating and traditional methods. Advances in Anatomic Pathology 1995;2:60-4

11 Erber WN, Gibbs TA, Ivey JG. Antigen retrieval by microwave oven heating for immunohistochemical analysis of bone marrow trephine biopsies. Pathology 1996;28:45-50.

12 Shi SR, Cote C, Kalra KL, Tandon AK. A technique for retrieving antigens in formalin-fixed, routinely acid decalcified, celloidin-embedded human temporal bone sections fied, celloidin-embedded human temporal bone sections for imm

13 Cattoretti G, Pileri S, Parravicini C, Becker MHG, Poggi S, Bifulco C, et al. Antigen unmasking on formalin-fixed paraffin embedded tissue sections. F Pathol 1993;171:83-98.

14 Cuevas EC, Bateman AC, Wilkins BS, Johnson PA Williams JH, Lee AHS, et al. Microwave antigen retrieval in
immunocytochemistry: a study of 80 antibodies. $\mathcal{F}$ Clin Pathol 1994;47:448-52.

15 Ho J, Schintacoo IP, Preston M, Said JW. Can microwave antigen retrieval replace frozen section immunohistochemistry in the phenotyping of lymphoid neoplasms? Appl Immunohistochem 1994;2:282-6.

16 Merz H, Malisius R, Mannweiler S, et al. A maximised immunohistochemical method for the retrieval and enhancement of hidden antigens. Lab Invest 1995;73:149-56.

17 Bobrow MN, Harris TD, Shaughnessy KJ, Litt DJ. Catalysed reporter deposition, a novel method of signal amplifications. Application to immunoassays. 7 Immunol Methods 1989;125:279-85.

18 Bobrow MN, Shaughnessy KJ, Litt DJ. Catalysed reporter deposition, a novel method of signal amplifications. II Application to membrane immunoassays. F Immunol Methods 1991;137:103-12.

19 Key ME, Phillips T. Catalysed signal amplification (CSA): An enhanced immunohistochemical staining method based on biotinyl tyramide. $₹$ NIH Research 1996;8:72-3.

20 Harris NL, Jaffe ES, Stein H, Banks PM, Chan JKC, Cleary $\mathrm{ML}$, et al. A revised European-American classification of lymphoid neoplasms: a proposal from the International Lymphoma Study Group. Blood 1994;84:1361-92.

21 General Haematology Task Force of the BCSH. Immunophenotyping in the diagnsosis of chronic lymphoprolifnophenotyping in the diagnsosis of chronic ly

22 Erber WN, Asbahr H, Rule SA, Scott CS. Unique immunophenotype of acute promyelocytic leukaemia as defined by $\mathrm{CD} 9$ and $\mathrm{CD} 68$ antibodies. $\mathrm{Br} \mathcal{F}$ Haemato 1994;88:101-4. 